\title{
EFFECTS OF SECONDARY TASK DEMANDS ON DRIVERS' RESPONSES TO EVENTS DURING DRIVING：SURROGATE METHODS AND ISSUES
}

\author{
Linda S. Angell \\ General Motors Corporation \\ Vehicle Structure \& Safety Integration Center \\ Warren, MI 48092 \\ E-mail: linda.s.angell@gm.com
}

PAPER NOT AVAILABLE

\begin{abstract}
\section{OBJECTIVES}

(1) To examine effects of secondary task demands on event detection and response during driving. (2) To examine the validity of surrogate methods which might be used in a laboratory environment to evaluate these effects early in the development process for advanced information systems. (3) To identify issues which limit and/or require careful interpretation in the application of event detection results to product considerations. (4) To identify issues which are at the forefront of scientific knowledge in this area, and in need of further research.
\end{abstract}

\section{METHODS}

Analytic methods were used to compare data derived from prior studies - and/or to make comparisons across studies. Linear mixed models, correlation, and other statistical methods were used, as appropriate. Results were drawn from several sources, and hence reflected multiple methodologies.

One of the main sources of data for this work was the CAMP Driver Workload Metrics project (2006) (which was a collaborative project between GM, Ford, Toyota, Nissan, and USDOT). Three types of methods were compared: on-road, test track, and laboratory methods. In the onroad and test-track methods, 108 participants and 69 participants (respectively) drove an instrumented vehicle that was the middle car in a platoon of three vehicles. (That is, the test participants followed a lead vehicle - and also had a vehicle following them). Drivers engaged in 22 different secondary tasks while driving on public roads (or a test track), and while also responding to three different types of visual events that could potentially occur during a task. These event types included: the illumination of a CHMSL-like light on the lead vehicle, a deceleration of the lead vehicle (without brake lights), and a turn signal illumination on the follow-vehicle.

In the laboratory, methods included a specially-developed variation of the Sternberg task, and two Peripheral Detection Tasks. Data were collected from 57 participants. Data from these methods are reported, compared with each other, and compared with data from other sources, where methods permitted that. Dependent variables were primarily "percent missed events"; key independent variables included task type and workload level. 


\section{RESULTS}

Data showed that visual-manual and auditory-vocal tasks differed in their effects on event detection during driving, with visual-manual tasks (as a class) interfering to a greater extent with event detection. The effects of task demands on event detection could not, however, be fully accounted for by glance-related measurements, suggesting that there are other variables involved, such as an attentional component to event detection. Furthermore, the modifiedSternberg method emerged as a laboratory surrogate with promising predictive validity (as well as repeatability) for evaluating a task's effects on event detection/response during driving. However, several issues emerged related to how the results of this assessment method can be interpreted and applied. These issues relate to practical matters (of task length, and task type), and also to theoretical matters (e.g., regarding how to change task design to mitigate interference with event detection).

\section{CONCLUSIONS}

Drivers' responses to events while driving (particularly unexpected events) are an important element of driving performance. Early in the development of advanced information systems, it would be desirable to have a valid way to evaluate how task demands may affect event detection (so that system task designs could be modified and improved, if necessary). However, it is particularly difficult to study event detection experimentally. Nearly all existing methods of evaluating event detection and response (other than naturalistic driving studies) must be classified as surrogate methods. And most of the methods examined here used techniques that did not really qualify as presenting "completely unexpected" events. Nonetheless, with this caveat, the method which offered the most promise was that which was sensitive both to a task's loading on visual processing and also its effects on central attention (e.g., to a cognitive/memory load). That method was the modified-Sternberg method.

Issues related to interpretation and application suggested that discriminating high from low workload tasks may be a problem when Task Types are separated. However, conclusions about grouping Task Types together are offered. Further, the findings had implications for strategies that might be used during system development to improve event detection performance for a task. Additional research is needed, not only to verify the kinds of strategies for task development that would in fact lead to improved event detection performance, but also to examine issues surrounding development of methods for presenting unexpected events, and to establish a deeper understanding of processes that underlie event detection and response, especially in naturalistic driving

\section{REFERENCES}

Angell, L. S., Auflick, L. L., Austria, P. A., Kochhar, D. S. \& Tijerina, L. (2006). "Driver workload metrics project: Task 2 final report," submitted to U.S. Dept. of Transportation, FHA, \& NHTSA under Cooperative Agreement Number DTFH61-01-X-00014. 